\title{
EL USO DE LOS VIDEOJUEGOS COMO HERRAMIENTA DIDÁCTICA PARA MEJORAR LA ENSEÑANZA-APRENDIZAJE: UNA REVISIÓN DEL ESTADO DEL TEMA
}

\section{The Use of Video Games as a Teaching Tool to Improve Teaching-Learning: State of the Art Review}

\author{
Angie Paola Roncancio-Ortiz ${ }^{1}$, Marco Fidel Ortiz-Carrera ${ }^{1}$, Humberto Llano-Ruiz ${ }^{1}$, Magally Jhoanna Malpica- \\ López ${ }^{1}$, D.E.A. José Joaquín Bocanegra-García² \\ ${ }^{1}$ Programa de Ingeniería de Sistemas, Universidad de la Amazonia (Florencia, Caquetá, Colombia). a.roncancio@udla. \\ edu.co,mar.ortiz@udla.edu.co, h.llano@udla.edu.co,m.malpica@udla.edu.co. \\ ${ }^{2}$ D.E.A., Pontificia Universidad Javeriana (Bogotá D.C., Colombia). \\ jose_bocanegra@javeriana.edu.co
}

(Recibido enero 2 de 2017 y Aprobado mayo 16 de 2017)

\begin{abstract}
Resumen
En esta nueva era del siglo XXI, cuando las sociedades están en constante cambio, la educación es el pilar para desarrollar no solo los conocimientos básicos y los instrumentos de cada una de las áreas del saber, sino también las habilidades que capaciten a la persona para desenvolverse en un mundo globalizado, en el que la comunicación y la socialización son herramientas fundamentales. En este escenario, los juegos de vídeo han pasado de ser simplemente un elemento de distracción para convertirse en valiosas herramientas, demostrando incluso que pueden desempeñar un papel protagónico en procesos relacionados con la educación, y como apoyo a las tareas docentes. Bajo esta premisa, el objetivo de este artículo es presentar una revisión sobre diversas experiencias particulares en las que se han incorporado videojuegos en las actividades de enseñanza y aprendizaje. Como resultado se evidencia que estas herramientas tecnológicas pueden ser utilizadas como mecanismos didácticos que ayudan, entre otras cosas, a la resolución de problemas de aprendizaje, al mejoramiento de las habilidades motoras y cognitivas, y al fomento de la creatividad.
\end{abstract}

Palabras clave: videojuegos educativos; enseñanza, aprendizaje; herramientas educativas; motivación.

\begin{abstract}
In this new era of the 21st century when societies are constantly changing, education is the cornerstone to provide the people not only with the basic knowledge and tools, but also with the required skills for facing the challenges in a globalized world. Thus, video games have evolved from distractive activities, to more valuable tools. These tools can play a leading role in education-related processes, for example, supporting teaching tasks. The aim of this article is to present a state of art review on several particular experiences in which video games have been incorporated into teaching and learning activities. As a result, video games can be used as didactic mechanisms that help students, among other things, to solve learning problems, to improve motor and cognitive skills, and to foster creativity.
\end{abstract}

Keywords: educational games; teaching; learning; educational tools; motivation.

\section{INTRODUCCIÓN}

Hoy más que nunca, la educación se constituye como un mecanismo primordial para fomentar no solo las nociones técnicas e instrumentales de cada una de las áreas del conocimiento, sino también para ayudar a desarrollar las destrezas que las personas requieren para desenvolverse en escenarios variantes. En ese sentido, la educación es un factor esencial en un mundo competitivo, globalizado e interconectado, donde las comunida-

Cómo citar este artículo:

A.P. Roncancio-Ortiz, M.F. Ortiz-Carrera, H. Llano-Ruiz, M.J. Malpica-Lopéz, J.J. Bocanegra García, “El uso de los videojuegos como herramienta didáctica para mejorar la enseñanza-aprendizaje: una revisión del estado del tema”, Revista Ingeniería Investigación y Desarrollo, vol. 17 (2), pp. 36-46, julio, 2017. 
des están expuestas a cambios constantes y requieren aprendizajes funcionales, activos y eficientes [1].

En los últimos años, los centros educativos han planteado nuevas metodologías, estrategias de enseñanza y políticas educativas, con el fin de facilitar y mejorar el aprendizaje de los estudiantes para que encaren los nuevos retos que impone una sociedad globalizada [2]. Una de esas estrategias son los videojuegos.

Los videojuegos son aplicaciones interactivas orientadas a la diversión que, a través de algunos mandos, posibilita simular experiencias en la pantalla de un televisor, una computadora u otro dispositivo electrónico [3]. Los videojuegos comparten varias similitudes con los juegos tradicionales y, en algunos casos, proporcionan beneficios adicionales como la interacción virtual con personas que se hallan en sitios diversos [4].

Estas y otras características hacen que los videojuegos sean adoptados como una herramienta que posibilita la circulación de los contenidos en los ambientes educativos tradicionales, convirtiéndose además, en una poderosa estrategia para mejorar la calidad de la educación [5].

Adicionalmente, cuando se usan los videojuegos no solo se pueden traer de vuelta al aula aspectos clave en el desarrollo de los estudiantes, como la diversión y la motivación, sino además fortalecer habilidades mentales y motoras. También se cree que el proceso de aprendizaje se hace más profundo que el tradicional, debido a la aplicación del principio denominado "aprender haciendo" [6].

Por tal motivo, cuando se usan los videojuegos como estrategia de aprendizaje, se espera que los estudiantes entiendan, asimilen y apropien con más facilidad las temáticas orientadas por los docentes [7].

Con base en lo anterior, este artículo presenta una revisión de literatura, en la que se analiza el uso de los videojuegos como una herramienta didáctica para mejorar el proceso de enseñanza-aprendizaje.

El artículo está dividido de la siguiente forma: la Sección II explica las consideraciones metodológicas del estudio; la Sección III describe brevemente las car- acterísticas de los videojuegos; la Sección IV analiza los videojuegos en la educación con sus usos particulares, tipos de videojuegos en la educación, habilidades y estilos de aprendizaje con los videojuegos, ventajas y desventajas. Finalmente, la Sección V expone las conclusiones.

\section{METODOLOGÍA}

Este trabajo se encuentra enmarcado dentro de la investigación denominada documental. Las ideas principales surgen del análisis de documentos, investigaciones y ponencias, que evidencian la importancia y la utilidad de los videojuegos en el campo de la educación.

La revisión de la literatura se hizo con el objetivo de seleccionar aquellos artículos que contenían información sobre el uso de los videojuegos como herramienta didáctica para mejorar la enseñanza-aprendizaje. Las fuentes principales de información fueron buscadores académicos especializados como Google Academic, Dialnet, Eric, Scielo, Worldwidescience y Redalyc.

La búsqueda inicial arrojó un resultado de 152 documentos, de los que se seleccionaron los 54 artículos más recientes y 13 documentos web como información complementaria. Como filtro adicional se estableció que el año de publicación fuera igual o superior al 2011.

\section{VIDEOJUEGOS}

Los juegos de computadora son definidos como "mundos virtuales con un conflicto" [8]. Los mundos virtuales son espacios de problemas finitos y basados en reglas que ofrecen a los jugadores diferentes medios para resolver problemas con un sistema preciso de retroalimentación y recompensa.

El proyecto denominado NIM, creado en 1951 para la computadora NIMROD, es considerado como uno de los primeros videojuegos [9]. Otros videojuegos similares a NIM, como Tennis for Two y Spacewar, surgieron en un entorno universitario y su aceptación por el público general derivó en que varios investigadores comenzaran a estudiar sus efectos sociales [10]. En 1969, Arpanet comienza a trabajar como red, lo que posibilita que una versión de Spacewar funcione en red [11]. 


\section{VIDEOJUEGOS EN LA EDUCACIÓN}

Al observar la cantidad de tiempo que los jóvenes pasan interactuando con sus ordenadores y dispositivos móviles, se concluyó que es posible aprovechar estos recursos y utilizarlos como una herramienta que ayude y permita a los estudiantes mejorar su aprendizaje [12].

Por eso, en los últimos años varias instituciones educativas han incorporado a sus currículos las tecnologías de la información y la comunicación (TIC), con el propósito de buscar nuevas estrategias que faciliten el aprendizaje y despierten el interés de los estudiantes en este [1314].

Mediante las TIC, los docentes pueden crear clases más amenas, que permitan explicar con facilidad temáticas complejas, asegurando la comprensión de los estudiantes [15-16].

Dentro de las TIC, un elemento para destacar son los videojuegos. Aunque estos tradicionalmente se han usado como un elemento de entretenimiento, en años recientes se han comenzado a incorporar en los procesos de enseñanza-aprendizaje [17].

Las investigaciones iniciales sobre videojuegos y enseñanza han sido amplias $y$, en general, se han hecho de manera descriptiva y poco concluyente [18]. Las primeras averiguaciones sobre juegos electrónicos son casi contemporáneas a la llegada de los primeros juegos de vídeos a los Estados Unidos, es decir, a inicios de los setenta [19]. No obstante, los primeros estudios que hablan de sus posibles impactos psicológicos y su potencial educativo para niños y adolescentes no saldrán en la literatura científica sino hasta diez años más tarde.

G. H. Ball es uno de los investigadores pioneros en este campo [20]. En su estudio titulado "Telegames Teach more than you Think", Ball propone dos líneas de investigación. Por una parte, el estudio del potencial de los videojuegos para desarrollar las habilidades especiales de los infantes, con gran interés en las apariencias tridimensionales y de representación de mundos reales. Por otra parte, la exploración de los elementos esenciales en el desarrollo intelectual de niños y adolescentes, como el lenguaje y las matemáticas. Ball analizó el papel ejercido por los videojuegos y en sus conclusiones enfatiza que los juegos electrónicos benefician destrezas intelectuales como la apropiación de conceptos numéricos, la interpretación y el estímulo de la lectura.

Otras investigaciones coinciden en señalar que los adolescentes con experiencia en videojuegos pueden incrementar sus capacidades espaciales, visuales y motoras, y que además los niños que utilizan videojuegos han logrado mejor rendimiento en pruebas sobre pensamiento crítico que los no jugadores [21].

En 1994, algunos autores [22] señalaron la necesidad de integrar los videojuegos al entorno educativo, no solo como un mecanismo de aprendizaje, sino como tema de análisis para determinar el impacto positivo en la educación con el desarrollo de capacidades cognitivas.

Sin embargo, para alcanzar resultados exitosos en el plano educativo es necesario contar con una planificación adecuada e implementar estrategias pertinentes que faciliten la comprensión de los contenidos por parte del estudiantado; todo lo anterior adaptado a un marco educativo que, fuera de exitoso, sea congruente con los objetivos pedagógicos, y que, además, mejore las competencias cognitivas [23-25].

De igual manera, los profesores que implementen los videojuegos en su plan de estudios necesitan bases tecnológicas sólidas para poder incorporar esas estrategias novedosas a los contenidos tradicionales del currículo [26]. Algunas competencias de los docentes que se requieren para este propósito son: el pensamiento crítico, la capacidad de resolución de problemas, el fomento de la creatividad y la innovación, y la habilidad de motivar a los estudiantes para que generen contenido.

\subsection{Tipos de videojuegos en la educación}

Un efecto adicional, dentro de la variedad que determina el fenómeno de los videojuegos, es el inconveniente para cualquier propósito de clasificación [27].

Una posible clasificación se puede hacer analizando el tipo de videojuego, por ejemplo, de acción, aventura, deportivo, de simulación, de rol y serio.

4.1.1 Videojuegos de acción (Arcades). Son juegos mediante los cuales no es necesario planificar una estrategia, sino que proponen una actividad para causar una respuesta precisa e interactuar con el entorno a través de acciones. Al incorporar videojuegos de acción en las 
aulas se mejora la atención, lo que permite a los alumnos dar respuestas más rápidas a los objetivos [28]. El aprendizaje se consigue por medio de acciones rápidas que aumentan la inmediata resolución de problemas.

4.1.2 Videojuegos de estrategia. El objetivo en estos videojuegos es planificar y establecer estrategias para avanzar por diferentes etapas. El alumno desarrolla el pensamiento lógico y la solución de problemas. El aprendizaje se obtiene mediante un entorno virtual, en el que se logra implicar a los jugadores para que participen en un sistema ordenado de relaciones que, en conjunto, conforman un contexto de interacción [29].

4.1.3 Videojuegos de aventura. La aventura es el elemento fundamental del juego, porque incorpora una alta interactividad. En este tipo de juegos se establece una trama narrativa y un conjunto de reglas que deben permitir la cohesión del relato, pero a su vez, aseguran cierto margen de libertad para que el jugador no se vea frustrado. También, ayudan al estudiante a desarrollar el pensamiento lógico y acrecientan la necesidad de tomar decisiones de forma constante [30]. El contenido de estos juegos puede ser utilizado como material por los docentes para ayudar a mejorar el desarrollo cognitivo.

4.1.4 Videojuegos deportivos. Los videojuegos deportivos/educativos pueden ser utilizados como herramientas para el aprendizaje del deporte. Motivan al estudiante a tener una vida activa. Su aplicación en los ámbitos de formación ha de ser contemplada de diferentes formas, en función del medio y las características del usuario. Generalmente, los jugadores simulan ser un deportista famoso, un personaje cuyos triunfos son vitoreados por quienes los observan [31].

4.1.5 Videojuegos de simulación. Estos videojuegos reproducen la realidad en forma ficticia, permiten estudiar y explorar el funcionamiento de máquinas, fenómenos y situaciones, y posibilitan aportar conocimientos específicos. El alumno construye su propio conocimiento en vez de recibirlo ya elaborado [32]. El aprendizaje se obtiene por medio de situaciones simuladas en las que hay que analizar estrategias de solución de problemas que el propio individuo decide y dirige [33].

4.1.6 Videojuegos de rol. Los videojuegos de rol muestran una gran variedad de opciones a los jugadores, quienes pueden crear su personaje, interactuar con otros integrantes, descubrir mundos virtuales, llevar a cabo acciones online y, al mismo tiempo, mejorar las habilidades con un constante aprendizaje de nuevas técnicas o aptitudes de su avatar (identidad virtual que representa al jugador en el videojuego) [34].

4.1.7 Videojuegos serios. Son juegos que no tienen entretenimiento, disfrute o diversión como su objetivo principal. El juego se basa en la idea de conectar un propósito serio a los conocimientos y las tecnologías [35].

\subsection{Habilidades y estilos de aprendizaje con los video- juegos}

En los últimos años, los conceptos de habilidades y estilos de aprendizaje han sido objeto de numerosas investigaciones, ya que permiten clasificar a los estudiantes en un conjunto de escalas de acuerdo con las formas en que reciben y procesan la información.

Al analizar el estilo de aprendizaje, el sistema de representación sensorial dominante y el perfil de estilos de pensamiento que tiene cada individuo, el docente es capaz de intervenir positivamente en el interés y la motivación de sus estudiantes, quienes al involucrarse más, van a mejorar su nivel de aprendizaje y su rendimiento académico [36-37].

Es por eso que al incorporar los videojuegos en la educación deben tenerse en cuenta los estilos de aprendizaje de los estudiantes [37].

Algunos de los estilos de aprendizaje que los docentes recientemente han comenzado a reconocer son [38]:

i. Visual (espacial): emplea imágenes, diagramas, colores y mapas mentales.

ii. Físico (kinestésico): usa su cuerpo, manos y sentido del tacto para ayudar en el aprendizaje.

iii. Aural (auditivo-musical): prefiere usar el sonido, el ritmo, la música, las grabaciones, entre otros.

iv. Verbal (lingüístico): usa palabras, tanto en el habla como en la escritura.

v. Lógico (matemático): prefiere usar la lógica y el razonamiento.

vi. Social (interpersonal): disfrutan aprendiendo en grupos o con otras personas.

vii. Solitario (intrapersonal): prefiere aprender solo y por medio del auto-estudio. 
Por las mismas características de los videojuegos, los estilos pueden sacar ventaja de su uso son el visual, el físico, el verbal, el lógico [39].
La Tabla 1 resume la correlación entre habilidades y estilos de aprendizaje que pueden ser abordados con ayuda de los videojuegos.

Tabla 1. Lista de algunas habilidades y estilos de aprendizajes

\begin{tabular}{ll}
\hline \multicolumn{1}{c}{ Estilos de aprendizaje } & \multicolumn{1}{c}{ Habilidades } \\
\hline Visual (espacial) & -Facilidad para absorber grandes cantidades de información con rapidez \\
& -Establecer relaciones entre distintas ideas y conceptos \\
& -Mejorar la memoria y la toma de decisiones \\
\hline Física (kinestésico) & -Mejorar las habilidades deportivas (reflejos, motricidad y coordinación) \\
& -Procesar la información asociándola a las sensaciones y movimientos \\
& -Participación activa \\
\hline Aural (auditivo-musical) & -Aumenta la atención \\
& -Mejora la organización \\
& -Mejora la comunicación \\
\hline Verbal (lingüístico) & -Aumenta la expresión oral \\
& -Promueve relaciones interpersonales \\
\hline Lógico (matemático) & -Produce una retención más duradera de la información \\
& -Ayuda al pensamiento lógico \\
\hline Social (interpersonal): & -Aumenta el análisis y el pensamiento crítico \\
& -Mejora la resolución de problemas \\
\hline Solitario (intrapersonal) & -Mejora el trabajo en equipo-Aumenta el liderazgo \\
& -Desarrolla valores como el respeto, la tolerancia, la solidaridad \\
\hline & -Formas sofisticadas de crítica, reflexión, observación y análisis \\
& -Intuición e independencia \\
\hline
\end{tabular}

Fuente: adaptado de [40]

4.3 Usos particulares de los videojuegos en la educación Con los avances de la tecnología, el uso de los videojuegos ha venido siendo aceptado en las aulas de clase, dado que estos generan en los estudiantes efectos perceptivos, cognitivos, conductuales, afectivos y motivacionales, lo que redunda en una mejora de la circulación del conocimiento y la comprensión del contenido curricular [41].
Además, el uso de los videojuegos en el aula puede complementar otros medios de enseñanza mediante las simulaciones, el uso de mundos virtuales y el desarrollo de cursos en línea [42].

La Tabla 2 detalla algunos videojuegos específicos y su aplicación en el aula.

Tabla 2. Lista de algunos videojuegos educativos y sus beneficios formativos

\begin{tabular}{lll}
\hline Juego & Desarrolladores/Editorial & Beneficios formativos \\
\hline Global Conflict: Palestine & Serious Games Interactive & Comprender el conflicto palestino \\
\hline Global Conflict: Latin America & Serious Games Interactive & $\begin{array}{l}\text { Conocer las causas y consecuencias de la contaminación } \\
\text { en América Latina }\end{array}$ \\
\hline Timez Attack & Big Brainz & Mejorar en álgebra \\
\hline Virtual Leader & Simulearn & Comprender el liderazgo \\
\hline 3D World Farmer & 3D World Farmer Team & Conocer las dificultades de los agricultores en África \\
\hline DimensionM & Tabula Digita & Mejorar en álgebra \\
\hline America's Army & UBI Soft & Entrenamiento militar \\
\hline
\end{tabular}




\begin{tabular}{|c|c|c|}
\hline Juego & Desarrolladores/Editorial & Beneficios formativos \\
\hline Darfur is Dying & $\begin{array}{l}\mathrm{mtvU} / \text { Reebok Human Rights Foun- } \\
\text { dation/International Crisis Group }\end{array}$ & $\begin{array}{l}\text { Conocer las condiciones de vida en los campos de refu- } \\
\text { giados de Darfur }\end{array}$ \\
\hline Food Force & $\begin{array}{l}\text { Deepend/Playerthree para el Pro- } \\
\text { grama Mundial de Alimentos de la } \\
\text { ONU }\end{array}$ & Ayuda humanitaria, gestión de alimentos y distribución \\
\hline Re-Mission & HopeLab & $\begin{array}{l}\text { Cómo ayudar y mejorar la vida de las personas con cán- } \\
\text { cer }\end{array}$ \\
\hline Revolution & The education Arcade & Aprender sobre la Revolución estadounidense \\
\hline SimPort & Tygron & $\begin{array}{l}\text { Dificultades de la construcción de grandes infraestruc- } \\
\text { turas }\end{array}$ \\
\hline Power Politics & Kellogg Creek Software/Cineplay & Procesos de una campaña presidencial en los EE.UU. \\
\hline Immune Attack & $\begin{array}{l}\text { Federation of American Scientists/ } \\
\text { Escape Hatch Entertainment }\end{array}$ & Funcionamiento del sistema inmunitario \\
\hline Missing & LiveWires Design & Navegar de forma segura en Internet \\
\hline Virtual U & Enlight Software & Conocer la gestión de la universidades estadounidenses \\
\hline DoomeD & $\begin{array}{l}\text { DESQ/University of Wolverhamp- } \\
\text { ton }\end{array}$ & Conocer la gestión de la universidades estadounidenses \\
\hline Cyber Budget (en francés) & $\begin{array}{l}\text { Paraschool/Ministerio de econo- } \\
\text { mía, finanzas e industria francés }\end{array}$ & Cómo gestionar las finanzas públicas \\
\hline
\end{tabular}

Fuente: adaptada de [43].

Como se observa en la Tabla 2, los usos de los videojuegos en la educación son variados. Estos van desde la compresión de la temática contemporánea (como el conflicto palestino) hasta el fortalecimiento de habilidades militares, por mencionar solo algunos de ellos.

\subsection{Ventajas de los videojuegos en la educación}

Algunas de las ventajas encontradas son el aumento de la creatividad, la facilidad del aprendizaje a través del descubrimiento, la asimilación y retención de la información, el mejoramiento de la coordinación motora y la capacidad de pensar y analizar rápidamente una situación, la ayuda en aspectos como la coordinación y la concentración en los detalles visuales, la exactitud, la velocidad de cálculo y la autoestima [44-47].

Los videojuegos también promueven el aprendizaje basado en la habilidad cognitiva, social y emocional, y una amplia gama de destrezas esenciales para el estudiante autónomo [44, 48, 49].

Además, si los videojuegos son aplicados en ambientes extraescolares donde los docentes hacen acompañamiento y asesoran a sus alumnos para su buen uso didáctico, se aumenta al máximo la oportunidad de que los niños sean activos, ya que estos juegos requieren de los jugadores que tengan una participación regular. Esto influye positivamente sobre la salud y el bienestar para los individuos y la sociedad en su conjunto [50]. Algunos autores [51] argumentan que los juegos pueden ser beneficiosos en otros dominios de bienestar personal y que además de ser mero disfrute, ayudan a los estudiantes en la generación de conocimiento y a mejorar académicamente [52], lo cual permite determinar que tanto el uso de la tecnología como de los juegos de vídeo puede ser un instrumento eficaz para el aprendizaje en los estudiantes [53].

Al adoptar este enfoque, se argumenta a favor de la importancia de evaluar la relación del estudiante con el videojuego [54], donde el resultado obtenido mediante encuestas muestra que estas metodologías pintan una imagen más clara y proporcionan conocimientos sobre el diseño de nuevas herramientas para enseñar a la próxima generación [55].

Ahora bien, vale la pena preguntarse si los videojuegos son realmente una buena opción para facilitar el desarrollo del aprendizaje de una manera eficaz y eficiente. Para ello, algunos autores [56] Ilevaron a cabo un estu- 
dio descriptivo sobre la nueva forma de implementar los videojuegos en los contenidos curriculares. En los resultados alcanzados determinaron que los estudiantes se sienten atraídos por este medio de enseñanza en comparación con la metodología tradicional, debido a que despierta el interés en la clase, haciéndola más interesante y participativa, permitiendo comprender con facilidad los temas impartidos en las aulas de clase, y logrando un impacto positivo en la solución de problemas de aprendizaje [57-59].

\subsection{Desventajas de los videojuegos en la educación} En la actualidad, la utilización de los videojuegos como herramienta de apoyo en la enseñanza ha despertado gran interés en la educación formal, haciendo que los docentes y profesores en formación se familiaricen con su uso [36]. Pero, en el contexto educativo un videojuego solo puede tener éxito cuando el contenido es adecuado, lo cual se verifica mediante pruebas de seguimiento de mejora continua de aprendizaje a los estudiantes [2]. No obstante, varios juegos usados en entornos educativos carecen de conexiones coherentes con las teorías del aprendizaje y del conocimiento [61].

Las posibles percepciones negativas de los padres son a menudo una barrera hacia la adopción de estas herramientas en los salones de clase. Los maestros, los estudiantes y la política de los fabricantes parecen estar influenciados por lo que los padres piensen acerca de la implementación de estas en el aula [62]. Esto se debe a que los padres menos experimentados se centran especialmente en los aspectos cuantitativos del uso de los videojuegos, como el número de horas que los estudiantes estarían jugando, lo cual puede causar adicción o conducta compulsiva a los jugadores, y ocasionar, por lo tanto, la interrupción de otras actividades educativas [63].

Por lo anterior, estas herramientas son a menudo vilipendiadas. Generalmente, los críticos citan las investigaciones sobre los efectos de los videojuegos considerados como violentos, mientras que los defensores, con frecuencia, mencionan investigaciones sobre la capacidad de percepción.

Lo interesante es que los puntos de vista de los críticos y los defensores son correctos acerca de los efectos que puedan tener los videojuegos [64]. Para que los videojuegos sean considerados por los padres y demás personas como una herramienta educativa viable deben proporcionar algún medio de prueba reconocible en el contexto educativo de la formación que se pretende impartir [5].

Sin embargo, en algunos casos, la aceptación de estos en el salón de clase sigue siendo limitada, debido a que hay docentes que no ven esta modalidad como una estrategia apta para la enseñanza [65-66].

Hay investigadores que indican que los videojuegos pueden ser perjudiciales para la salud de los niños. Dentro de sus consecuencias se pueden destacar la adicción, las crisis convulsivas en el $1 \%$ de los niños con epilepsia fotosensible, la estimulación de actitudes violentas que afectan las actividades cotidianas, la falta de atención, el aislamiento e, incluso, la inflamación de los tendones de la mano (nintendinitis). Además, el uso excesivo de los videojuegos puede generar trastornos como dolor de cabeza, irritación de los ojos, dolores musculares y malas posturas, que están ligados directamente con el tiempo empleado en el juego [67].

\section{CONCLUSIONES}

En este artículo se ha presentado una revisión sobre diversas experiencias particulares, en las que se han incorporado videojuegos en las actividades de enseñanza y aprendizaje.

En términos generales, el resultado del análisis evidencia que los videojuegos son herramientas aptas para la resolución de problemas de aprendizaje, el mejoramiento de habilidades motoras y cognitivas, el fomento de la creatividad, entre otras.

A pesar de esto, también se comprueba que el uso de los videojuegos puede acarrear problemas tanto comportamentales como de salud. Algunos de estos son la adicción a los videojuegos, el estímulo hacia actitudes violentas, la inflamación de los tendones de la mano (nintendinitis), los dolores de cabeza, los dolores musculares y la irritación de los ojos.

A pesar de los inconvenientes que pueden generar, consideramos que sus ventajas están por encima de las desventajas. 


\section{REFERENCIAS}

[1] B. Sampedro Requena y K. McMullin, "Videojuegos para la inclusión educativa," Digital Education Review, n. 27, pp. 122-137, 2015 Doi: http://dx.doi. org/10.6018/j/149121.

[2] F. De Grove, J. Van Looy y J. Bourgonjon, "Digital games in the classroom? A contextual approach to teachers' adoption intention of digital games in formal education", Computers in Human Behavior, vol. 28, n.o 6, pp. 2023-2033, 2012. doi:https:// doi.org/10.1016/j.chb.2012.05.021

[3] J. Pérez Porto \& A. Gardey. (2013). Videojuego. [En línea]. Disponible: http://definicion.de/videojuego/

[4] I. Granic, A. Lobel y R. Engels, "The Benefits of Playing Video Games", American Psychologist, vol. 69, n.o 1, pp. 66-78, 2014. doi: https://doi. org/10.1037/a0034857

[5] F. Bellotti, B. Kapralos, K. Lee, P. Moreno-Ger y R. Berta, "Assessment in and of Serious Games: An Overview," Advances in Human-Computer Interaction, vol. 2013, n.o 1, pp. 1-11, 2013. doi: http:// dx.doi.org/10.1155/2013/136864.

[6] A. Nordby, K. Øygardslia, U. Sverdrup y H. Sverdrup. (2016). The art of Gamification; Teaching Sustainability and System Thinking by Pervasive Game Development, The Electronic Journal of e-Learning. [En línea]. 14, (3), 152-168, 2016 Disponible en: http://files.eric.ed.gov/fulltext/EJ1107121.pdf.

[7] J.-L. Senger y R. Kanthan, "The impact of specially designed digital games-based learning in undergraduate pathology and medical education", Specially Designed Digital Games Based Learning in Pathology, vol. 135, n.o 1, pp. 135-142, 2011. Doi: 10.1043/2009-0698-OAR1.1.

[8] S. Egenfeldt-Nielsen, "What makes a good Learning game?: going beyond edutainment", Elearn Magazine, vol. 2011, n.o 2, 2011. doi: doi:https:// doi.org/10.1145/1943208.1943210

[9] J. L. Gómez Eguia, R. S. Contreras Espinosa y L. Solano Albajes, "Videojuegos: conceptos, historia y su potencial como herramientas para la educación," 3 c TIC: Cuadernos de desarrollo aplicados a las TIC 1.2, vol. 1, n.o 2, pp. 1-14, 2012. doi:http:// dx.doi.org/10.17993/3ctic.2012.12.

[10] M. Trenta, "Orígenes del videojuego: conexiones históricas y sociales de un producto cultural". Presentado en IV Congreso Internacional Latina de Comunicación , Laguna, 2012.
[11] I. B. Antonio. (2015). La carrera docente centra el debate educativo ante el 20-D, Andalucíaeduca. [En línea]. 8(165), 9-12. Disponible: http://www. andaluciaeduca.com/hemeroteca/ae_digital165. pdf

[12] M. Ružić-Baf, H. Strnak y A. Debeljuh, “Online Video Games and Young People", International Journal of Research in Education and Science, vol. 2, n. 1, pp. 93-103, 2016. doi: http://dx.doi.org/10.21890/ ijres.19610.

[13] S. Martin, G. Diaz, E. Sancristobal, R. Gil, M. Castro y J. Peire, "New technology trends in education: Seven years of forecasts and convergence", Computers \& Education, vol. 57, n. 3, pp. 1893-1906, 2011. doi: https://doi.org/10.1016/j.compedu.2011.04.003

[14] B. Gros y A. Forés, "Dues tendències TAP en educació. Apoderament i participació mitjançant les tecnologies", Revista de Psicologia, Ciències de l'Educació i de l'Esport, vol. 31, n.o 1, pp. 83-88, 2013. Disponible: http://diposit.ub.edu/dspace/ bitstream/2445/104301/1/627661.pdf.

[15] S. Egenfeldt-Nielsen. (2011). Los videojuegos como herramienta de aprendizaje. [En línea]. Disponible: https://tecnoeducativas.files.wordpress. com/2011/03/egenfeld-nielsen-los-videojuegos-como-herramientas-de-aprendizaje.pdf

[16] A. Berns, A. Gonzalez Pardo y D. Camacho, "Game-like language learning in 3-D virtual environments", Computers \& Education, vol. 60, n. 1, p. 210-220, 2013. doi: https://doi.org/10.1016/j. compedu.2012.07.001

[17] P. Backlund y M. Hendrix, "Educational games - are they worth the effort? A literature survey of the effectiveness of serious games", presentado In Games and Virtual Worlds for Serious Applications (VS-GAMES) 5th International Conference on IEEE, Bournemouth, 2013. doi: https://doi.org/10.1109/ vs-games. 2013.66

[18] A. Mesa Méndez y C. J. Burgos Dávila. (2012). Jóvenes y videojuegos: preocupaciones y vivencias. Un análisis de las prácticas cotidianas al videojugar. Revista Argentina de Estudios de Juventud. [En línea]. 1(5), 1-36. Disponible en: http://perio.unlp. edu.ar/ojs/index.php/revistadejuventud/article/ view/1503/1274.

[19] L. Anyó. (2013). Género y cultura global en el videojuego español. Pasavento: Revista de Estudios Hispánicos. [En línea]. 1(2), 247-263. Disponible: http://dspace.uah.es/dspace/handle/10017/24101?show=full. 
[20] M. de Aguilera y A. Méndiz. (s.f.). Un balance de la investigación sobre videojuegos: análisis de efectos y valoración de su capacidad educativa. [En línea]. Disponible: https://www.academia.edu/8373773/ Un_balance_de_la_investigaci\%C3\%B3n_sobre_ videojuegos_An\%C3\%A1lisis_de_efectos_y_valoraci\%C3\%B3n_de_su_capacidad_educativa.

[21] K. V. Rodríguez. (s.f.). Videojuegos y salud. Un campo de investigación fértil. [En línea]. Disponible: http://www.espididoctor.com/videojuegos-salud-investigacion/

[22] A. Méndiz, J. Pindado, J. Ruiz y J. M. Pulido. (s.f.). Videojuegos y educación: revisión crítica de la investigación realizada. [En línea]. Disponible: http://ares.cnice.mec.es/informes/02/documentos/iv04_0301a.htm

[23] G.-J. Hwang, H.-Y. Sung, C.-M. Hung, L.-H. Yang y I. Huang, "A knowledge engineering approach to developing educational computer games for improving students' differentiating knowledge", British Journal of Educational Technology, vol. 44, n.ㅇ 2, pp. 183-196, 2012.

doi:https://doi.org/10.1111/j.1467-8535.2012.01285.x

[24] C. Linehan, B. Kirman, S. Lawson y G. G. Chan, "Practical, Appropriate, Empirically-Validated Guidelines for Designing Educational Games", Presented In Proceedings of the SIGCHI Conference on Human Factors in Computing Systems, Vancouver, 2011. Doi: https://doi.org/10.1145/1978942.1979229

[25] W.-H. Wu, H.-C. Hsiao, P.-L. Wu, C.-H. Lin y S.-H. Huang, "Investigating the learning-theory foundations of game-based learning: a meta-analysis", Journal of Computer Assisted Learning, vol. 28, n.으 3, p. 265-279, 2012 doi: https://doi.org/10.1111/ j.1365-2729.2011.00437.x.

[26] R. Kenny y G. Gunter. (2011). Factors Affecting Adoption of Video Games in the Classroom. Journal of Interactive Learning Research. [En línea]. 22(2), 259-276. Disponible: https://eric.ed.gov/?i$d=E J 937028$.

[27] M. Rubio Méndez. (2012). Retos y posibilidades de la introducción de videojuegos en el aula. Revista de Estudios de Juventud. [En línea]. (98), 118-134. Disponible: http://www.injuve.es/sites/default/files/2012/46/publicaciones/Revista98_9.pdf.

[28] H. G. Rodríguez Celis y M. Sandoval Escobar. (2011). Consumo de videojuegos y juegos para computador: influencias sobre la atención, memoria, rendimiento académico y problemas de conducta. Suma Psicológica. [En línea]. 18(2), 99-
110. Disponible: http://www.redalyc.org/articulo. oa?id=134222985008.

[29] J. M. Garrido Miranda. (2013). Videojuegos de estrategia: algunos principios para la enseñanza. Revista Electrónica de Investigación Educativa. [En línea]. 15(1), 62-74. Disponible: http://www.redalyc.org/articulo.oa?id=15528262005.

[30] S. Gómez García y N. Navarro Sierra, "Videojuegos e Información. Una aproximación a los newsgames españoles como nueva óptica informativa," Icono 14, vol. 11, n. 2, pp. 31-51, 2013. doi: https://doi. org/10.7195/ri14.v11i2.604.

[31] A. E. Garduño Sánchez y L. A. Garduño Sánchez. (2011). Estereotipos corporales en las portadas de los videojuegos de género deportivo. Revista Internacional de Medicina y Ciencias de la Actividad Física y el Deporte. [En línea]. 11(42), 407-420. Disponible: http://cdeporte.rediris.es/revista/revista42/artcuerpo213.htm.

[32] F. I. Revuelta Domínguez y J. Guerra Antequera. (2012). ¿Qué aprendo con videojuegos? Una perspectiva de metaaprendizaje del videojugador. RED. Revista de Educación a Distancia. [En línea]. 15(33), 10. Disponible: http://www.redalyc.org/ articulo.oa?id=54724495006.

[33] C. González Tardón y M. Calvo Alonso. (2015). Aportaciones desde el diseño de videojuegos y la simulación para la mejora de proyectos formativos basados en Realidad Virtual y videojuegos serios. Comunicación y Pedagogía: Nuevas Tecnologías y Recursos didácticos. [En línea]. (287), 54-60. Disponible: https://dialnet.unirioja.es/servlet/articulo? codigo $=5390537$.

[34] C. Marco y M. Chóliz, "Tratamiento cognitivo-conductual de la adicción a videojuegos de rol online: fundamentos de propuesta de tratamiento y estudio de caso", Anales de Psicología, vol. 30, n.o 1, pp. 46-55, 2014. doi: http://dx.doi.org/10.6018/ analesps.30.1.150851.

[35] D. Djaouti, J. Alvarez y J. P. Jessel, "Classifying Serious Games: the G/P/S model", in Handbook of Research on Improving Learning and Motivation through Educational Games: Multidisciplinary Approaches. London: Patrick Felicia (ed), 2011. doi: https://doi.org/10.4018/978-1-60960-495-0. ch006

[36] M. C. V. Hincapié. (2014). Relación entre los estilos de aprendizaje y los niveles de creatividad motriz en los estudiantes de la institución educativa las delicias del municipio de El Bagre. [En línea]. Disponible: http://bibliotecadigital.udea.edu.co/bits- 
tream/10495/2774/1/1.\%20PROYECTO\%20MAESTR\%C3\%8DA\%20EN\%20EDUCACI\%C3\%93N\%20 FINAL.pdf.

[37] E. D. Mosquera. (2012). Estilos de aprendizaje. Revista EIDOS. [En línea]. (5), 5-11. Disponible: https://www.ute.edu.ec/posgrados/eidos5/art-1. html.

[38] J. Ferriman. (2013). 7 major-learning styles which one is you. [En línea]. Disponible: https://www. learndash.com/7-major-learning-styles-whichone-is-you/.

[39] J. Feldman, A. Monteserin y A. Aman. (2012). Juegos digitales para la detección del estilo de percepción de los estudiantes. En 13th Argentine Symposium on Artificial Intelligence, ASAI 2012. [En línea]. Disponible: http://41jaiio.sadio.org.ar/ sites/default/files/10_ASAI_2012.pdf.

[40] Learning-styles-online.com (2017). Learning-styles-online.com. [En línea]. Disponible: https://www.learning-styles-online.com/overview/.

[41] T. M. Connolly, E. A. Boyle, E. MacArthur, T. Hainey y J. M. Boyle, "A systematic literature review of empirical evidence on computer games and serious games", Computers \& Education, vol. 58, n.으 2, p. 661-686, 2012 doi: https://doi.org/10.1016/j. compedu.2012.03.004

[42] D. Short. (2012). Teaching scientific concepts using a Virtual World Minecraft. Teaching Science. [En línea]. 58(3), 55-58. Disponible: https://www. academia.edu/1891072/Teaching_Scientific_Concepts_Using_a_Virtual_World_-_Minecraft.

[43] P. Felicia. (2009, jun.). Videojuegos en el aula. [En línea]. Disponible: http://games.eun.org/upload/ GIS_HANDBOOK_ES.pdf.

[44] E. Koh, B. Wadhwa, Y. Gee Kin y J. Lim, "Teacher Perceptions of Games in Singapore Schools", Simulation \& Gaming, vol. 43, n.. 1, pp. 51-66, 2012 doi: https://doi.org/10.1177/1046878111401839.

[45] J. M. Muñoz González, S. Rubio García y I. Cruz Pichardo. (2015). Strategies of Collaborative Work in the Classroom Through the Design of Video Games. Digital Education Review. [En línea]. 27, 6984. Disponible: http://revistes.ub.edu/index.php/ der/article/view/11914.

[46] L. Miller, C. Chang, S. Wang, M. Beier y Y. Klisch, "Learning and motivational impacts of a multimedia science game", Computers \& Education, vol. 57, n. 1, p. 1425-1433, 2011. doi: https://doi.org/10.1016/j.compedu.2011.01.016.

[47] B. G. Salvat. (2014). Análisis de las prestaciones de los juegos digitales para la docencia universitaria.
Revista Interuniversitaria de Formación del Profesorado. [En línea]. 79(28.1), 115-128, Disponible: https://www.academia.edu/7669063/An\%C3\%A1 lisis_de_las_prestaciones_de_los_juegos_digitales_para_la_docencia_universitaria?auto=download.

[48] E. Marchetti y A. Valente, "Learning via Game Design: From Digital to Card Games and Back Again," Electronic Journal of e-Learning, vol. 13, № 3, pp. 167-180, 2015 Disponible en: http://files.eric.ed. gov/fulltext/EJ1060177.pdf.

[49] J. M. Sáez López, J. Mille, E. Vázquez Cano y M. C. Domínguez Garrido. (2015). Exploring Application, Attitudes and Integration of Video Games: MinecraftEdu in Middle School. Educational Technology \& Society. [En línea]. 18(3), 114-128. Disponible: https://ssrn.com/abstract=2700646.

[50] J. Eun Lee, C. Huang, Z. Pope y Z. Gao. (2015). Integration of Active Video Games in Extracurricular Activity at Schools. JTRM in Kinesiology. [En línea]. (1), 1-10, 2015 Disponible: https://eric.ed.gov/?i$d=E J 1053416$.

[51] J. Bourgonjon, G. Vandermeersche, B. De Wever, R. Soetaert y M. Valcke, "Players' perspectives on the positive impact of video games: A qualitative content analysis of online forum discussions", New Media \& Society, vol. 18, n.o 8, p. 1732-1749, 2016. doi: https://doi.org/10.1177/1461444815569723.

[52] M. N. Giannakos, "Enjoy and learn with educational games: Examining factors affecting learning performance", Computers \& Education, vol. 68, pp. 429-439, 2013 doi. https://doi.org/10.1016/j.compedu.2013.06.005.

[53] N. Shin, L. M. Sutherland, C. A. Norris y E. Soloway, "Effects of game technology on elementary student learning in mathematics", British Journal of Educational Technology, vol. 43, n.o 4, p. 540560, 2012. doi:https://doi.org/10.1111/j.14678535.2011.01197.x.

[54] G. Van Eaton, D. B. Clark y B. E. Smith, "Patterns of Physics Reasoning in Face-to-Face and Online Forum Collaboration Around a Digital Game", International Journal of Education in Mathematics, Science and Technology, vol. 3, n. 1, pp. 1-13, 2015. doi: http://dx.doi.org/10.18404/ijemst.10740.

[55] D. Weintrop y U. Wilensky, "Situating Programming Abstractions in a Constructionist Video Game", Informatics in Education, vol. 13, n.o 2, p. 307-321, 2014. doi: http://dx.doi.org/10.15388/ infedu.2014.10. 
[56] V. Marín, M. López y G. Maldonado. (2015). Can Gamification be introduced within primary classes? Digital Education Review. [En línea]. (27), 5568. Disponible: http://revistes.ub.edu/index.php/ der/article/view/11913.

[57] D. Eseryel, V. Law, D. Ifenthaler, X. Ge y R. Miller. (2014). An Investigation of the Interrelationships between Motivation, Engagement, and Complex Problem Solving in Game-based Learning. Educational Technology \& Society. [En línea]. 17(1), 42-53. Disponible: http://www.ifets.info/journals/17_1/5.pdf.

[58] N. Padilla Zea, F. Gutiérrez, J. R. López Arcos, A. Abad Arranz y P. Paderewski. (2014). Modeling storytelling to be used in educational video games. Computers in Human Behavior. [En línea]. 31, 461-474. doi: https://doi.org/10.1016/j.chb.2013.04.020.

[59] W. R. Watson, C. J. Mong y C. A. Harris. "A case study of the in-class use of a video game for teaching high school history", Computers \& Education, vol. 56, n.o 2, pp. 466-474, 2011 doi: http://doi.org/10.1016/j.compedu.2010.09.007.

[60] N. Padilla Zea, C. A. Collazos Ordoñez, F. L. Gutiérrez Vela y N. Medina Medina, "Videojuegos educativos: Teorías y propuestas para el aprendizaje en grupo", Ciencia e Ingeniería Neogranadina, vol. 22, n. 1, pp. 139-150, 2012. doi: http://dx.doi. org/10.18359/rcin.254.

[61] C. Kinzer, D. Hoffman, S. Türkay, P. Chantes, T. Dvorkin, A. Chaiwinij y N. Günba. (2012). The Impact of Choice and Feedback on Learning, Motivation, and Performance in an Educational Video Game. Proceedings of the Games, Learning, and Society Conference. [En línea]. 2, 175-181. Disponible: https://
www.academia.edu/2993997/The_Impact_of_ Choice_and_Feedback_on_Learning_Motivation_ and_Performance_in_an_Educational_Video_ Game.

[62] J. Bourgonjon, M. Valcke, R. Soetaert, B. de Wever y T. Schellens, "Parental acceptance of digital game-based learning", Computers \& Education, vol. 57, n.o 1, p. 1434-1444, 2011 Doi: https://doi.org/10.1016/j.compedu.2010.12.012.

[63] H. Friedrichs, F. von Gross, K. Herde y U. Sander. (2015). Parents' Views of Video Games: Habitus Forms in the Context of Parental Mediation. Journal of Media Literacy Education. [En línea]. 7(1), 58-64. Disponible: https://eric.ed.gov/?i$\mathrm{d}=$ EJ1074688.

[64] D. Gentile, "The Multiple Dimensions of Video Game Effects", Child Development Perspectives, vol. 5, n.o 2, pp. 75-81, 2011 doi: https://doi.org/10.1111/j.1750-8606.2011.00159.x.

[65] T. N. Nguyen, "Motivational Effect of Web-Based Simulation Game in Teaching Operations Management", Journal of Education and Training Studies, vol. 3, n. 2, pp. 9-15, 2015. doi: http://dx.doi. org/10.11114/jets.v3i2.565.

[66] B. S. Jong, C. H. Lai, Y. T. Hsia, T. W. Lin y C. Y. Lu, "Using game-based cooperative learning to improve learning motivation: A study of online game use in an operating systems course", IEEE Transactions on Education, vol. 56, n. 2, pp. 183-190, 2013. doi: https://doi.org/10.1109/TE.2012.2207959.

[67] A. Serrano y R. Espinoza. (s.f.). ¿Qué es importante saber acerca de los videojuegos? [En línea]. Disponible: https://www.aciprensa.com/recursos/los-video-juegos-465/. 